\title{
OTHER FIXED POINT THEOREMS FOR TWO PAIRS OF ABSORBING MAPPINGS SATISFYING AN IMPLICIT RELATION IN PARTIAL METRIC SPACES
}

\author{
V. Popa, A.-M. Patriciu
}

ABstract. In this paper some fixed point theorems for two pairs of absorbing mappings satisfying an implicit relation are proved, which extend Theorem 5 [21] to two pairs of mappings. As application, a general fixed point theorem for a sequence of mappings in partial metric spaces is obtained.

2010 Mathematics Subject Classification: 54H25, 47H10.

Keywords: partial metric space, fixed point, absorbing mapping, implicit relation.

\section{INTRODUCTION}

In 1994, Matthews [15] introduced the concept of partial metric space as a part of the study of denotational semantics of dataflow networks and proved the Banach contraction principle in such spaces.

The partial metric spaces play an important role in construction models in theory of computation.

Many authors studied the fixed points for mappings satisfying some contractive condition in partial metric spaces.

Quite recently, in [2], [3], [11] some fixed point theorems under various contractive conditions are proved. In [12] and [13] some fixed point theorems for pairs of mappings in partial metric spaces are proved. Other results are obtained in [1].

In 1994, Pant [17] introduced the notion of pointwise $R$ - weak commutativity which is equivalent to commutativity in coincidence points. Jungck [10] defined $f$ and $g$ to be weak compatible if $f x=g x$ implies $f g x=g f x$.

Then, $f$ and $g$ are weakly compatible if and only if $f$ and $g$ are pointwise $R$ weakly commuting.

In [12] some fixed point theorems for weakly compatible mappings in partial metric spaces are proved. 
V. Popa, A.-M. Patriciu - Other fixed point theorems for two pairs of ...

The notion of pointwise absorbing mappings is introduced and studied in [4] [6].

Some results for two pairs of absorbing mappings are obtained in [14], [16].

Several classical fixed point theorems and common fixed point theorems have been unified considering a general condition by an implicit relation in [18], [19]. Recently, the method is used in the study of fixed points in metric spaces, symmetric spaces, quasi - metric spaces, $b$ - metric spaces, ultra - metric spaces, convex metric spaces, Hilbert spaces, compact metric spaces, in two and three metric spaces, for single valued mappings, hybrid pairs of mappings and set - valued mappings.

Quite recently, the method is used in the study of fixed points of mappings satisfying contractive / extensive conditions of integral type, in fuzzy metric spaces, probabilistic metric spaces, intuitionistic metric spaces, $G$ - metric spaces, $G_{p}$ metric spaces. With this method the proofs of some fixed points theorems are more simple.

Also, the method allows the study of local and global properties of fixed point structures.

The study of fixed points for mappings in complete partial metric spaces satisfying implicit relations is initiated in [8], [9], [20] - [22].

The purpose of this paper is to prove some fixed point theorems for two pairs of pointwise absorbing mappings satisfying an implicit relation in complete partial metric spaces, generalizing Theorem 2.2 [13]. As application, a general fixed point theorem for a sequence of mappings in partial metric spaces is obtained.

\section{Preliminaries}

Definition 1 ([15]). Let $X$ be a nonempty set. A function $p: X \times X \rightarrow \mathbb{R}_{+}$is said to be a partial metric on $X$ if for any $x, y, z \in X$, the following conditions hold:

$\left(P_{1}\right): p(x, x)=p(y, y)=p(x, y)$ if and only if $x=y$,

$\left(P_{2}\right): p(x, x) \leq p(x, y)$,

$\left(P_{3}\right): p(x, y)=p(y, x)$,

$\left(P_{4}\right): p(x, z) \leq p(x, y)+p(y, z)-p(y, y)$.

The pair $(X, p)$ is called a partial metric space.

If $p(x, y)=0$, then $x=y$, but the converse does not always hold.

Each partial metric $p$ on $X$ generates a $T_{0}$ - topology $\tau_{p}$ which has as a base the family of open $p$ - balls $\left\{B_{p}(x, \varepsilon): x \in X, \varepsilon>0\right\}$, where $B_{p}(x, y)=\{y \in X$ : $p(x, y) \leq p(x, x)+\varepsilon\}$ for all $x \in X$ and $\varepsilon>0$.

A sequence $\left\{x_{n}\right\}$ in a partial metric space $(X, p)$ converges to a point $x \in X$ 
V. Popa, A.-M. Patriciu - Other fixed point theorems for two pairs of ...

with respect to $\tau_{p}$ if and only if

$$
p(x, x)=\lim _{n \rightarrow \infty} p\left(x_{n}, x\right) .
$$

If $p$ is a partial metric on $X$, then the function $p^{s}(x, y)=2 p(x, y)-p(x, x)-p(y, y)$ defines a metric on $X$. if

Furthermore, a sequence $\left\{x_{n}\right\}$ converges in $\left(X, p^{s}\right)$ to a point $x \in X$ if and only

$$
\lim _{m, n \rightarrow \infty} p\left(x_{n}, x_{m}\right)=\lim _{n \rightarrow \infty} p\left(x_{n}, x\right)=p(x, x) .
$$

Definition 2. Let $(X, p)$ be a partial metric space.

a) A sequence $\left\{x_{n}\right\}$ in $X$ is said to be a Cauchy sequence if $\lim _{n, m \rightarrow \infty} p\left(x_{n}, x_{m}\right)$ exists and is finite.

b) $\quad(X, p)$ is said to be complete if every Cauchy sequence $\left\{x_{n}\right\}$ in $X$ converges with respect to $\tau_{p}$ to a point $x \in X$ such that $\lim _{n \rightarrow \infty} p\left(x_{n}, x\right)=p(x, x)$.

Lemma 1 ([3], [15]). Let $(X, p)$ be a partial metric space. Then:

a) A sequence $\left\{x_{n}\right\}$ in $X$ is a Cauchy sequence in $(X, p)$ if and only if $\left\{x_{n}\right\}$ is a Cauchy sequence in $\left(X, p^{s}\right)$.

b) A partial metric space $(X, p)$ is complete if and only if the metric space $\left(X, p^{s}\right)$ is complete.

Lemma 2 ([3], [1]). Let $(X, p)$ be a partial metric space and $\left\{x_{n}\right\}$ be a sequence in $X$. If $\lim _{n \rightarrow \infty} x_{n}=x$ and $p(x, x)=0$, then

$$
\lim _{n \rightarrow \infty} p\left(x_{n}, y\right)=p(x, y), \forall y \in X .
$$

Theorem 3 (Theorem $2.2[13])$. Suppose that $(X, p)$ is a complete partial metric space and $T, S$ are self mappings on $X$. If there exists $r \in[0,1)$ such that

$$
p(T x, S y) \leq r M(x, y),
$$

for any $x, y \in X$, where

$$
M(x, y)=\max \left\{p(x, y), p(x, T x), p(y, S y), \frac{1}{2}[p(x, S y)+p(y, T x)]\right\},
$$

then there exists $z \in X$ such that $z=T z=S z$.

Definition $3([4],[5])$. 1) Let $(X, d)$ be a metric space and $f, g$ be two self mappings on $X$. $f$ is said to be $g$ absorbing if there exists $R>0$ such that

$$
d(g x, g f x) \leq R d(g x, f x)
$$


V. Popa, A.-M. Patriciu - Other fixed point theorems for two pairs of ...

for all $x \in X$.

2) $f$ is said to be pointwise $g$ absorbing if for given $x \in X$, there exists $R>0$ such that

$$
d(g x, g f x) \leq R d(g x, f x) .
$$

Similarly we can define $f$ absorbing and pointwise $f$ absorbing map.

Remark 1. If $(X, p)$ is a partial metric space we have similar definition of Definition 3 with $p$ instead $d$.

Remark 2. If $g$ is $I$ - identity map in $X$, then $f$ is trivial absorbing. Similarly, $I$ is $f$ absorbing in respect of any $f$.

\section{IMPLICIT RELATIONS}

Definition 4. Let $\mathcal{F}_{5}$ be the set of all continuous functions $F: \mathbb{R}_{+}^{5} \rightarrow \mathbb{R}$ satisfying:

$\left(F_{1}\right): F$ is nonincreasing in variable $t_{5}$,

$\left(F_{2}\right):$ There exists $h \in[0,1)$ such that for all $u, v \geq 0$,

$\left(F_{2 a}\right): F(u, v, v, u, u+v) \leq 0$

and

$\left(F_{2 b}\right): F(u, v, u, v, u+v) \leq 0$

implies $u \leq h v$,

$\left(F_{3}\right): F(t, t, 0,0,2 t)>0, \forall t>0$.

In the following examples, property $\left(F_{1}\right)$ is obviously.

Example 1. $F\left(t_{1}, \ldots, t_{5}\right)=t_{1}-k \max \left\{t_{2}, t_{3}, t_{4}, \frac{t_{5}}{2}\right\}$, where $k \in[0,1)$.

$\left(F_{2}\right):$ Let $u, v \geq 0$ be such that

$$
F(u, v, v, u, u+v)=u-k \max \left\{u, v, \frac{u+v}{2}\right\} \leq 0 .
$$

If $u>v$, then $u(1-k) \leq 0$, a contradiction. Hence, $u \leq v$, which implies $u \leq h v$, where $0 \leq h=k<1$.

Similarly, $F(u, v, u, v, u+v) \leq 0$ implies $u \leq h v$.

$\left(F_{3}\right): F(t, t, 0,0,2 t)=t(1-k)>0, \forall t>0$.

Example 2. $F\left(t_{1}, \ldots, t_{5}\right)=t_{1}-k \max \left\{t_{2}, t_{3}, t_{4}, t_{5}\right\}$, where $k \in\left[0, \frac{1}{2}\right)$.

The proof is similar to the proof of Example 1. 
V. Popa, A.-M. Patriciu - Other fixed point theorems for two pairs of ...

Example 3. $F\left(t_{1}, \ldots, t_{5}\right)=t_{1}-\frac{a t_{3} t_{1}}{1+t_{2}}-b t_{2}-c\left(t_{3}+t_{4}\right)-d t_{5}$, where $a, b, c, d \geq 0$ and $a+b+2 c+2 d<1$.

$\left(F_{2}\right):$ Let $u, v \geq 0$ be such that

$$
F(u, v, v, u, u+v)=u-\frac{a u v}{u+v}-c(u+v)-d(u+v) \leq 0 .
$$

If $u>v$, then $u[1-(a+b+2 c+2 d)] \leq 0, a$ contradiction. Hence, $u \leq v$, which implies $u \leq h v$, where $0 \leq h=a+b+2 c+2 d<1$.

Similarly, $F(u, v, u, v, u+v) \leq 0$ implies $u \leq h v$.

$\left(F_{3}\right): F(t, t, 0,0,2 t)=t[1-(b+2 d)]>0, \forall t>0$.

Example 4. $F\left(t_{1}, \ldots, t_{5}\right)=t_{1}-a \max \left\{t_{2}, t_{3}, t_{4}\right\}-b t_{5}$, where $a, b \geq 0$ and $a+2 b<1$.

Example 5. $F\left(t_{1}, \ldots, t_{5}\right)=t_{1}^{2}-a t_{2} t_{3}-b t_{3} t_{4}-c t_{5}^{2}$, where $a, b, c \geq 0$ and $a+b+4 c<1$.

Example 6. $F\left(t_{1}, \ldots, t_{5}\right)=t_{1}^{2}+\frac{t_{1}}{1+t_{5}}-\left(a t_{2}^{2}+b t_{3}^{2}+c t_{4}^{2}\right)$, where $a, b, c \geq 0$ and $a+b+c<1$.

Example 7. $F\left(t_{1}, \ldots, t_{5}\right)=t_{1}-a t_{2}-b t_{3}-c \max \left\{2 t_{4}, t_{5}\right\}$, where $a, b, c \geq 0$ and $a+b+2 c<1$.

Example 8. $F\left(t_{1}, \ldots, t_{5}\right)=t_{1}^{2}-t_{1}\left(a t_{2}+b t_{3}+c t_{4}\right)-d t_{5}^{2}$, where $a, b, c, d \geq 0$ and $a+b+c+4 d<1$.

\section{MAIN RESUlts}

Theorem 4. Let $(X, p)$ be a partial metric space and $A, B, S, T$ be self mappings on $X$ such that for all $x, y \in X$

$$
F\left(\begin{array}{c}
p(A x, B y), p(S x, T y), p(S x, A x) \\
p(T y, B y), p(S x, B y)+p(A x, T y)
\end{array}\right) \leq 0
$$

where $F \in \mathcal{F}_{5}$. If $z$ is a common fixed point of $A, B, S$ and $T$, then $p(z, z)=0$.

Proof. Suppose that $x=y=z$. Then by (2) we obtain

$$
F(p(z, z), p(z, z), p(z, z), p(z, z), 2 p(z, z)) \leq 0,
$$

a contradiction of $\left(F_{3}\right)$ if $p(z, z)>0$. Hence, $p(z, z)=0$.

Theorem 5. Let $A, B, S$ and $T$ be self mappings of a partial metric space $(X, p)$ as in Theorem 4. Then $A, B, S$ and $T$ have at most one common fixed point. 
V. Popa, A.-M. Patriciu - Other fixed point theorems for two pairs of ...

Proof. Suppose that $u, v$ are common fixed points of $A, B, S$ and $T$. Then by (2) we have

$$
F(p(u, v), p(u, v), p(u, u), p(v, v), p(u, v)+p(u, v)) \leq 0 .
$$

By Theorem $4, p(u, u)=p(v, v)=0$. Hence,

$$
F(p(u, v), p(u, v), 0,0,2 p(u, v)) \leq 0,
$$

a contradiction of $\left(F_{3}\right)$ if $p(u, v)>0$. Hence, $p(u, v)=0$, which implies $u=v$.

Theorem 6. Let $(X, p)$ be a complete partial metric space and $A, B, S$ and $T$ be self mappings on $X$ such that

1) $\quad A(X) \subset T(X)$ and $B(X) \subset S(X)$,

2) the inequality (2) holds for all $x, y \in X$ and some $F \in \mathcal{F}_{5}$.

If one of $A(X), B(X), S(X)$ or $T(X)$ is closed, then:

1) $A$ and $S$ have a coincidence point,

2) $B$ and $T$ have a coincidence point.

Moreover, if $A$ is pointwise $S$ absorbing and $B$ is pointwise $T$ absorbing, then $A, B, S$ and $T$ have a unique common fixed point.

Proof. Let $x_{0}$ be an arbitrary point in $X$. Since $A(X) \subset T(X)$, there exists $x_{1} \in X$ such that $T x_{1}=A x_{0}$.

Since $B(X) \subset S(X)$, there exists $x_{2} \in X$ such that $S x_{2}=B x_{1}$. Continuing this process we can construct sequences $\left\{x_{n}\right\}$ and $\left\{y_{n}\right\}$ in $X$ such that

$$
y_{2 n}=T x_{2 n+1}=A x_{2 n}, y_{2 n+1}=S x_{2 n+2}=B x_{2 n+1} .
$$

We prove that $\left\{y_{n}\right\}$ is a Cauchy sequence in $(X, p)$.

By (2) for $x=x_{2 n}$ and $y=x_{2 n+1}$ we have

$$
F\left(\begin{array}{c}
p\left(A x_{2 n}, B x_{2 n+1}\right), p\left(S x_{2 n}, T x_{2 n+1}\right), p\left(S x_{2 n}, A x_{2 n}\right), \\
p\left(T x_{2 n+1}, B x_{2 n+1}\right), p\left(S x_{2 n}, B x_{2 n+1}\right)+p\left(A x_{2 n}, T x_{2 n+1}\right)
\end{array}\right) \leq 0 .
$$

By (3) we obtain

$$
F\left(\begin{array}{l}
p\left(y_{2 n}, y_{2 n+1}\right), p\left(y_{2 n-1}, y_{2 n}\right), p\left(y_{2 n-1}, y_{2 n}\right), \\
p\left(y_{2 n}, y_{2 n+1}\right), p\left(y_{2 n-1}, y_{2 n+1}\right)+p\left(y_{2 n}, y_{2 n}\right)
\end{array}\right) \leq 0 .
$$

By $\left(P_{4}\right)$,

$$
p\left(y_{2 n-1}, y_{2 n+1}\right) \leq p\left(y_{2 n-1}, y_{2 n}\right)+p\left(y_{2 n}, y_{2 n+1}\right)-p\left(y_{2 n}, y_{2 n}\right) .
$$

By $(4)$ and $\left(F_{1}\right)$ we have

$$
F\left(\begin{array}{l}
p\left(y_{2 n}, y_{2 n+1}\right), p\left(y_{2 n-1}, y_{2 n}\right), p\left(y_{2 n-1}, y_{2 n}\right), \\
p\left(y_{2 n}, y_{2 n+1}\right), p\left(y_{2 n-1}, y_{2 n}\right)+p\left(y_{2 n}, y_{2 n+1}\right)
\end{array}\right) \leq 0 .
$$


V. Popa, A.-M. Patriciu - Other fixed point theorems for two pairs of ...

By $(5)$ and $\left(F_{2 a}\right)$ we obtain

$$
p\left(y_{2 n}, y_{2 n+1}\right) \leq h p\left(y_{2 n-1}, y_{2 n}\right) .
$$

Similarly by (2) for $x=x_{2 n+2}$ and $y=x_{2 n+1}$ we have

$$
F\left(\begin{array}{c}
p\left(A x_{2 n+2}, B x_{2 n+1}\right), p\left(S x_{2 n+2}, T x_{2 n+1}\right), p\left(S x_{2 n+2}, A x_{2 n+2}\right), \\
p\left(T x_{2 n+1}, B x_{2 n+1}\right), p\left(S x_{2 n+2}, B x_{2 n+1}\right)+p\left(A x_{2 n+2}, T x_{2 n+1}\right)
\end{array}\right) \leq 0 .
$$

By (3) we have

$$
F\left(\begin{array}{c}
p\left(y_{2 n+2}, y_{2 n+1}\right), p\left(y_{2 n+1}, y_{2 n}\right), p\left(y_{2 n+1}, y_{2 n+2}\right), \\
p\left(y_{2 n}, y_{2 n+1}\right), p\left(y_{2 n+1}, y_{2 n+1}\right)+p\left(y_{2 n+2}, y_{2 n}\right)
\end{array}\right) \leq 0 .
$$

By $\left(P_{4}\right)$,

$$
p\left(y_{2 n}, y_{2 n+2}\right) \leq p\left(y_{2 n}, y_{2 n+1}\right)+p\left(y_{2 n+1}, y_{2 n+2}\right)-p\left(y_{2 n+1}, y_{2 n+1}\right) .
$$

By $(6)$ and $\left(F_{1}\right)$ we have

$$
F\left(\begin{array}{c}
p\left(y_{2 n+2}, y_{2 n+1}\right), p\left(y_{2 n+1}, y_{2 n}\right), p\left(y_{2 n+1}, y_{2 n+2}\right), \\
p\left(y_{2 n}, y_{2 n+1}\right), p\left(y_{2 n}, y_{2 n+1}\right)+p\left(y_{2 n+1}, y_{2 n+2}\right)
\end{array}\right) \leq 0
$$

By $\left(F_{2 b}\right)$ we have

$$
p\left(y_{2 n+2}, y_{2 n+1}\right) \leq h p\left(y_{2 n}, y_{2 n+1}\right) .
$$

Hence

$$
p\left(y_{n}, y_{n+1}\right) \leq h p\left(y_{n-1}, y_{n}\right) \leq \ldots \leq h^{n} p\left(y_{0}, y_{1}\right) .
$$

For $n, m \in \mathbb{N}$ with $m>n$ and $\left(P_{4}\right)$ we obtain

$$
\begin{aligned}
p\left(y_{n}, y_{m}\right) & \leq\left(h^{n}+h^{n+1}+\ldots+h^{m-1}\right) p\left(y_{0}, y_{1}\right) \\
& \leq \frac{h^{n}}{1-h} p\left(y_{0}, y_{1}\right)
\end{aligned}
$$

and so

$$
p^{s}\left(y_{n}, y_{m}\right) \leq 2 p\left(y_{n}, y_{m}\right) \rightarrow 0 \text { as } n, m \rightarrow \infty .
$$

This implies that $\left\{y_{n}\right\}$ is a Cauchy sequence in the metric space $\left(X, p^{s}\right)$. Since $(X, p)$ is complete, then by Lemma $1,\left(X, p^{s}\right)$ is complete. Therefore, there exists $y \in X$ such that $\lim _{n \rightarrow \infty} p^{s}\left(x_{n}, y\right)=0$ and by $(1), p(y, y)=\lim _{n \rightarrow \infty} p\left(y_{n}, y_{n}\right)=$ $\lim _{n, m \rightarrow \infty} p\left(y_{n}, y_{m}\right)=0$. This implies

$$
\lim _{n \rightarrow \infty} p\left(y_{2 n+1}, y\right)=\lim _{n \rightarrow \infty} p\left(y_{2 n-1}, y\right) \text { and } \lim _{n \rightarrow \infty} p\left(S x_{2 n}, y\right)=0 .
$$


V. Popa, A.-M. Patriciu - Other fixed point theorems for two pairs of ...

Now we suppose without loss of generality that $S(X)$ is a closed subset of partial metric space $(X, p)$.

Then, there exists $u \in X$ such that $y=S u$.

By (2) for $x=u$ and $y=x_{2 n+1}$ we obtain

$$
\begin{gathered}
F\left(\begin{array}{c}
p\left(A u, B x_{2 n+1}\right), p\left(S u, T x_{2 n+1}\right), p(S u, A u), \\
p\left(T x_{2 n+1}, B x_{2 n+1}\right), p\left(S u, B x_{2 n+1}\right)+p\left(A u, T x_{2 n+1}\right)
\end{array}\right) \leq 0 \\
F\left(\begin{array}{c}
p\left(A u, y_{2 n+1}\right), p\left(S u, y_{2 n}\right), p(S u, A u), \\
p\left(y_{2 n}, y_{2 n+1}\right), p\left(S u, y_{2 n+1}\right)+p\left(A u, y_{2 n}\right)
\end{array}\right) \leq 0 .
\end{gathered}
$$

Letting $n$ tend to infinity by (7) and Lemma 2 we obtain

$$
F(p(A u, y), 0, p(y, A u), 0,0+p(A u, y)) \leq 0 .
$$

By $\left(F_{2 b}\right)$ we have $p(y, A u)=0$ which implies $y=A u=S u$.

Since $A(X) \subset T(X), y \in T(X)$, hence, there exists $v \in X$ such that $y=T v$. By (2) for $x=u$ and $y=v$ we obtain

$$
\begin{gathered}
F\left(\begin{array}{c}
p(A u, B v), p(S u, T v), p(S u, A u), \\
p(T v, B v), p(S u, B v)+p(A u, T v)
\end{array}\right) \leq 0, \\
F(p(y, B v), 0,0, p(y, B v), p(y, B v)+0) \leq 0 .
\end{gathered}
$$

By $\left(F_{2 a}\right), p(y, B v)=0$ which implies $y=B v=T v$. Hence, $y=A u=S u=$ $T v=B v$ with $p(y, y)=0$.

If $A$ is pointwise $S$ absorbing, there exists $R>0$ such that

$$
p(S u, S A u) \leq R p(S u, A u)=R p(y, y)=0 .
$$

Hence,

$$
S u=S A u=S S u=y
$$

which implies

$$
y=S y .
$$

Now we prove that $y=A y$. By (2) we have

$$
\begin{gathered}
F\left(\begin{array}{c}
p\left(A y, B x_{2 n+1}\right), p\left(S y, T x_{2 n+1}\right), p(S y, A y), \\
p\left(T x_{2 n+1}, B x_{2 n+1}\right), p\left(S y, B x_{2 n+1}\right)+p\left(A y, T x_{2 n+1}\right)
\end{array}\right) \leq 0, \\
F\left(\begin{array}{c}
p\left(A y, y_{2 n+1}\right), p\left(x, y_{2 n}\right), p(y, A y), \\
p\left(y_{2 n}, y_{2 n+1}\right), p\left(y, y_{2 n+1}\right)+p\left(A y, y_{2 n}\right)
\end{array}\right) \leq 0 .
\end{gathered}
$$


V. Popa, A.-M. Patriciu - Other fixed point theorems for two pairs of ...

Letting $n$ tend to infinity by Lemma 2 we obtain

$$
F(p(A y, y), 0, p(y, A y), 0,0+p(A y, y)) \leq 0 .
$$

Hence, by $\left(F_{2 b}\right)$ we obtain $p(y, A y)=0$, which implies $y=A y$ and $y$ is a common fixed point of $A$ and $S$.

If $B$ is pointwise $T$ absorbing, there exists $R_{1}>0$ such that

$$
p(T v, T B v) \leq R_{1} p(T v, B v)=R_{1} p(y, y)=0 .
$$

Hence,

$$
T v=T B v=T T v=y
$$

which implies

$$
y=T y
$$

By (2) we have

$$
\begin{gathered}
F\left(\begin{array}{c}
p(A u, B y), p(S u, T y), p(S u, A u), \\
p(T y, B y), p(S u, B y)+p(A u, T y)
\end{array}\right) \leq 0, \\
F(p(y, B y), 0,0, p(y, B y), p(y, B y)+0) \leq 0 .
\end{gathered}
$$

By $\left(F_{2 a}\right)$ we obtain $p(y, B y)=0$, which implies $y=B y$ and $y$ is a common fixed point of $B$ and $T$.

Hence, $y$ is a common fixed point of $A, B, S$ and $T$. By Theorem $5, y$ is the unique common fixed point of $A, B, S$ and $T$.

Example 9. Let $X=[0,1]$ be a partial metric space with $p(x, y)=\max \{x, y\}$. Then $(X, p)$ is a complete partial metric space. We define the following mappings on $X$.

$A x=0, S x=\frac{x}{x+1}, B x=\frac{x}{3}, T x=x$. Then $A(X)=\{0\}, S(X)=\left[0, \frac{1}{2}\right]$, $B(X)=\left[0, \frac{1}{3}\right], T(X)=[0,1]$. Hence, $A(X) \subset T(X), B(X) \subset S(X)$ and $B(X)$, $S(X), T(X)$ are closed subsets of $(X, p)$. Moreover,

$$
\begin{gathered}
p(S x, S A x)=p\left(\frac{x}{x+1}, 0\right)=\frac{x}{x+1}, \\
p(S x, A x)=\frac{x}{x+1} .
\end{gathered}
$$

Hence,

$$
p(S x, S A x) \leq R p(S x, A x) \text { for some } R \geq 1
$$


V. Popa, A.-M. Patriciu - Other fixed point theorems for two pairs of ...

$$
p(T x, T B x)=\max \left\{x, \frac{x}{3}\right\}=x .
$$

Hence,

$$
p(T x, T B x) \leq R_{1} p(T x, B x) \text { for some } R_{1} \geq 1 .
$$

Hence, $A$ is pointwise $S$ absorbing and $B$ is pointwise $T$ absorbing. On the other hand,

$$
\begin{aligned}
& p(A x, B y)=p\left(0, \frac{y}{3}\right)=\frac{y}{3} \\
& p(T y, B y)=p\left(y, \frac{y}{3}\right)=y
\end{aligned}
$$

Hence,

$$
p(A x, B y) \leq k p(T y, B y)
$$

where $k \in\left[\frac{1}{3}, \frac{1}{2}\right)$, which implies

$$
p(A x, B y) \leq k \max \{p(S x, T y), p(S x, A x), p(T y, B y), p(S x, B y)+p(T y, A x)\} .
$$

By Theorem 6, A,B, S and T have a unique common fixed point.

If $S=T=I$, by Theorem 6 and Remark 2 we obtain

Theorem 7. Let $(X, p)$ be a complete partial metric space and $A, B$ be self mappings on $X$ such that the inequality

$$
F\left(\begin{array}{c}
p(A x, B y), p(x, y), p(x, A x), \\
p(y, B y), p(x, B y)+p(y, A x)
\end{array}\right) \leq 0
$$

holds for all $x, y \in X$ and some $F$ satisfying properties $\left(F_{1}\right),\left(F_{2 a}\right)$ and $\left(F_{3}\right)$. Then $A$ and $B$ have a unique common fixed point.

Remark 3. By Theorem 7 and Example 1 we obtain Theorem 3.

For a function $f:(X, p) \rightarrow(X, p)$ we denote

$$
F i x(f)=\{x \in X: x=f x\} .
$$

If instead of $\left(F_{1}\right)$ we have property

$\left(F_{1}^{\prime}\right): F$ is nonincreasing in variables $t_{2}, t_{3}, t_{4}, t_{5}$, we have 
V. Popa, A.-M. Patriciu - Other fixed point theorems for two pairs of ...

Theorem 8. Let $A, B, S$ and $T$ be self mappings of a partial metric space $(X, p)$. If the inequality (2) holds for all $x, y \in X$ and some $F$ satisfying properties $\left(F_{1}^{\prime}\right),\left(F_{2}\right)$ and $\left(F_{3}\right)$, then we have:

$$
[F i x(S) \cap F i x(T)] \cap F i x(A)=[F i x(S) \cap F i x(T)] \cap F i x(B) .
$$

Proof. Let $x \in[F i x(S) \cap F i x(T)] \cap F i x(A)$. Then by (2) we have

$$
\begin{gathered}
F\left(\begin{array}{c}
p(A x, B x), p(S x, T x), p(S x, A x), \\
p(T x, B x), p(S x, B x)+p(A x, T x)
\end{array}\right) \leq 0, \\
F(p(x, B x), p(x, x), p(x, x), p(x, B x), p(x, B x)+p(x, x)) \leq 0 .
\end{gathered}
$$

By $\left(F_{2}\right)$,

$$
p(x, x) \leq p(x, B x)
$$

and by $\left(F_{1}^{\prime}\right)$ we obtain

$$
F(p(x, B x), p(x, B x), p(x, B x), p(x, B x), 2 p(x, B x)) \leq 0,
$$

which implies by $\left(F_{2}\right)$ that

$$
p(x, B x) \leq h p(x, B x) .
$$

Hence, $p(x, B x)=0$, so $x=B x$ and $x \in F i x(B)$. Therefore,

$$
[F i x(S) \cap F i x(T)] \cap F i x(A) \subset[F i x(S) \cap F i x(T)] \cap F i x(B) .
$$

Similarly,

$$
[F i x(S) \cap F i x(T)] \cap F i x(B) \subset[F i x(S) \cap F i x(T)] \cap F i x(A) .
$$

By Theorems 6 and 6 we obtain

Theorem 9. Let $S, T$ and $\left\{A_{i}\right\}_{i \in \mathbb{N}^{*}}$ be self mappings of a complete partial metric space such that:

a) $\quad A_{2}(X) \subset S(X)$ and $A_{1}(X) \subset T(X)$,

b) one of $A_{1}(X), A_{2}(X), S(X), T(X)$ is a closed subset of $X$,

c) the inequality

$$
F\left(\begin{array}{c}
p\left(A_{i} x, A_{i+1} y\right), p(S x, T y), p\left(S x, A_{i} x\right), \\
p\left(T y, A_{i+1} y\right), p\left(S x, A_{i+1} y\right)+p\left(T y, A_{i} x\right)
\end{array}\right) \leq 0,
$$

holds for all $x, y \in X, i \in \mathbb{N}^{*}$ and $F$ satisfies conditions $\left(F_{1}^{\prime}\right),\left(F_{2}\right)$ and $\left(F_{3}\right)$.

If $A_{1}$ is $S$ pointwise absorbing and $A_{2}$ is pointwise $T$ absorbing, then $S, T$ and $\left\{A_{i}\right\}_{i \in \mathbb{N}^{*}}$ have a unique common fixed point. 
V. Popa, A.-M. Patriciu - Other fixed point theorems for two pairs of ...

\section{REFERENCES}

[1] T. Abdeljawad, W. Karapinar and K. Tas, Existence and uniqueness of a common fixed point on partial metric spaces, Appl. Math. Lett. 24 (11) (2011), 1900-1904.

[2] I. Altun, F. Sola and H. Simsek, Generalized contractive principle on partial metric spaces, Topology Appl. 157 (18) (2010), 2778-2785.

[3] R. Chi, E. Karapinar and T. D. Thanh, A generalized contraction principle in partial metric spaces, Math. Comput. Modelling 55 (5 - 6) (2012), 1673-1681.

[4] D. Gopal, A. S. Ranadive and U. Mishra, On some open problems of common fixed point theorems of noncompatible self maps, Proc. Math. BHU 20 (2004), 135-141.

[5] D. Gopal, A. S. Ranadive and R. P. Pant, Common fixed points for absorbing maps, Bull. Marathwada Math. Soc. 9 (1) (2008), 43-48.

[6] D. Gopal, M. Imdad, M. Husain and D. K. Patel, Proving common fixed point theorems for Lipschitzian type mappings via absorbing pairs, Bull. Math. Anal. Appl. 3 (4) (2011), 92-100.

[7] D. Gopal, M. Hasan and M. Imdad, Absorbing pairs faciliting common fixed point theorems for Lipschitzian type mappings in symmetric spaces, Commun. Korean Math. Soc. 27 (2) (2012), 385-397.

[8] S. Guliaz and E. Karapinar, Common limit points in partial metric spaces through implicit functions, Math. Statist. 42 (4) (2013), 347-353.

[9] S. Guliaz, E. Karapinar and I. S. Yuce, A coupled common fixed point theorem in partial metric space with an implicit relation, Fixed Point Theory Appl. 2013, 2013:38.

[10] G. Jungck, Common fixed points for noncontinuous nonself maps on nonnumeric spaces, Far East J. Math. Sci. 4 (2) (1991), 195-215.

[11] Z. Kadelburg, H. K. Nashine and S. Radenović, Fixed point results under various contractive conditions in partial metric spaces, Rev. R. Acad. Cienc. Exactas Fis. Nat., Ser. A Mat., RACSAM 10 (2013), 241-256.

[12] E. Karapinar and I. M. Erhan, Fixed point theorems for operators on partial metric spaces, Appl. Math. Lett. 24 (2011), no. 11, 1900-1904.

[13] E. Karapinar and U. Yüksel, Some common fixed point theorems in partial metric spaces, J. Appl. Math. 2011 (2011), Article ID 263621, 16 pages.

[14] E. Karapinar, D. K. Patel, M. Imdad and D. Gopal, Some nonunique common fixed point theorems in symmetric spaces through $C L R_{(S T)}$ - property, Intern. J. Math. Math. Sci. 2013 (2013), Article ID 753965, 8 pages.

[15] S. G. Matthews, Partial metric topology and applications, Proc. 8th Summer Conference on General Topology and Applications, Ann. New York Acad. Sci. 728 (1994), 183-197. 
V. Popa, A.-M. Patriciu - Other fixed point theorems for two pairs of ...

[16] U. Mishra, R. P. Pathak, Rashmi, Common fixed point of absorbing mappings satisfying Lipschitzian type contractive condition, Intern. J. Pure Appl. Math. 77 (2) (2012), 245-253.

[17] R. P. Pant, Common fixed points for noncommuting mappings, J. Math. Anal. Appl. 188 (1994), 436-440.

[18] V. Popa, Fixed point theorems for implicit contractive mappings, Stud. Cercet. Ştiint., Ser. Mat., Univ. Bacău 7 (1997), 129-133.

[19] V. Popa, Some fixed point theorems for compatible mappings satisfying an implicit relation, Demonstr. Math. 32, 1 (1999), 157-163.

[20] V. Popa and A.-M. Patriciu, A general fixed point theorem for a pair of self mappings with common limit range property in partial metric spaces, Bul. Inst. Politehn. Iaşi, Ser. Mat. Mec. Teor. Fiz. 61 (65) (2015), 85-99.

[21] V. Popa and A.-M. Patriciu, A general fixed point theorem for a pair of mappings in partial metric spaces, Acta Univ. Apulensis, Math. Inform. 43 (2015), 93-103.

[22] C. Vetro and F. Vetro, Common fixed points of mappings satisfying implicit relations in partial metric spaces, J. Nonlinear Sci. Appl. 6 (2013), 152-161.

Valeriu Popa

"Vasile Alecsandri" University of Bacău,

Bacău, Romania

email:vpopa@ub.ro

Alina-Mihaela Patriciu

Department of Mathematics and Computer Sciences,

Faculty of Sciences and Environment,

"Dunărea de Jos" University of Galaţi,

Galaţi, Romania

email: Alina.Patriciu@ugal.ro 